\title{
Endoscopic therapy using novel fan devices
}
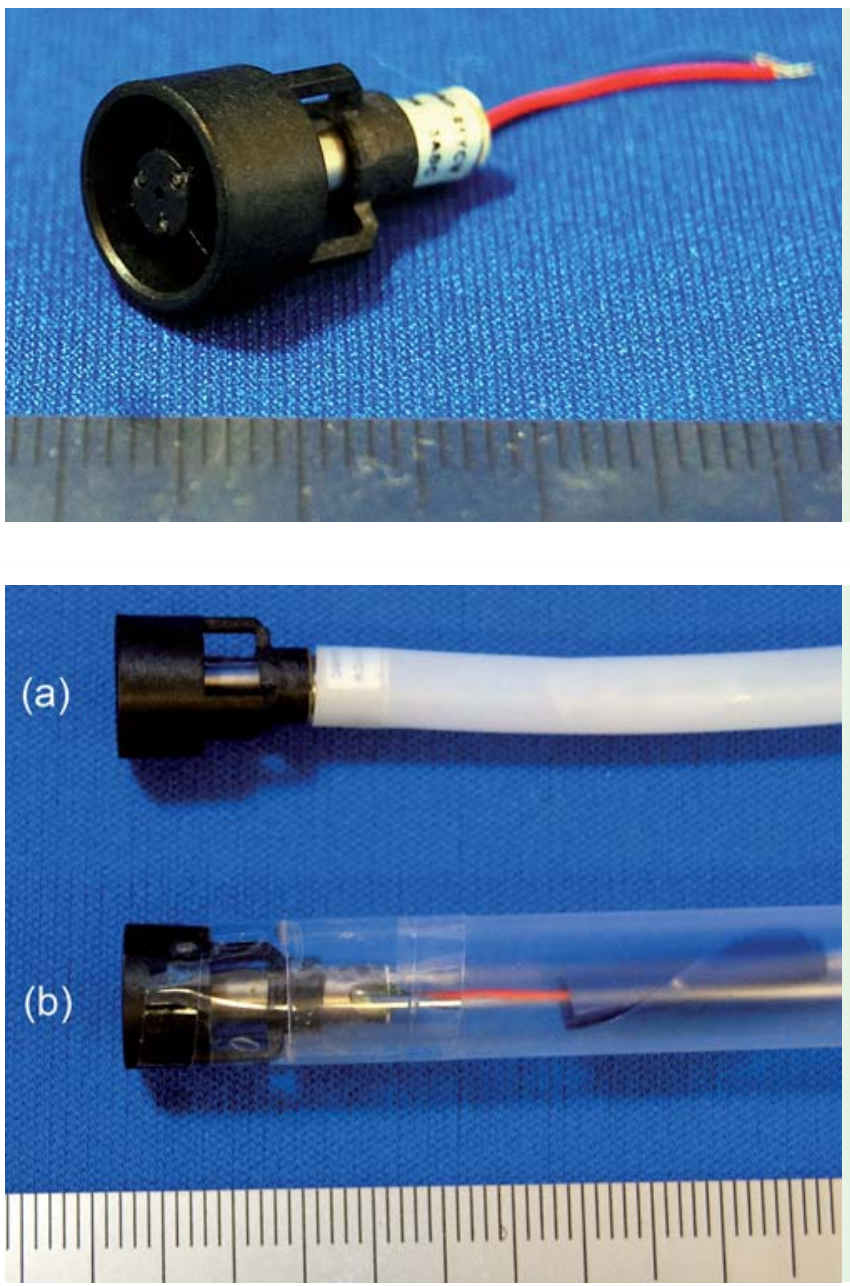

Fig. 2 Endoscopic fan

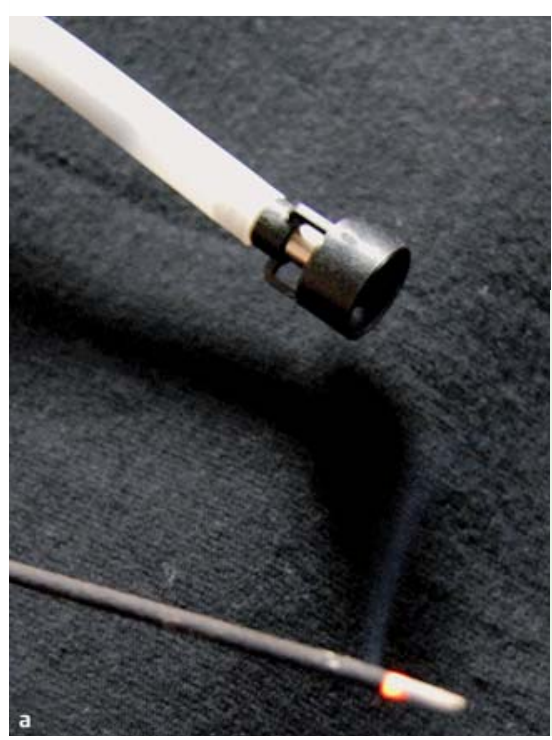

Fig. 1 Supermicro fan motor. device: a blowing type; and $\mathbf{b}$ ventilation type.

During the use of electrocautery in endoscopic procedures such as incision, dissection, and resection, smoke can accumulate in the gastrointestinal tract, impairing the visual field, which causes problems in

continuing the procedure. This problem is usually resolved by aspirating all of the air in the gastrointestinal tract along with the smoke and replaced with it with new air, thereby improving the visual field. However, this has to be done several times, which prolongs the procedure.

In this article, we describe the use of novel fan devices that we have developed to enable improving the visual field by only blowing the air rather than changing the air in the gastrointestinal tract. Both devices have been created using a supermicro fan motor (Shiko Inc., Kanagawa, Japan) (৫ Fig. 1).

The first, blowing-type, device works by blowing the air, whereas the second is a ventilation-type device ( $\mathbf{F i g} . \mathbf{2}$ ).

- Fig. 3 illustrates how the two devices work, using an incense stick to produce the smoke.

To evaluate the usefulness of the devices in endoscopy, we used them during experimental endoscopic submucosal dissection (ESD) in resected porcine stomachs ( $\bullet$ Fig. 4).

This involved injecting $6 \mathrm{~mL}$ of physiological saline to the submucosa, and use of a constant $(200 \mathrm{~W})$ high-frequency power source (UES-20, Olympus, Tokyo, Japan) for electrocautery. The procedure was conducted both with and without the use of the fan devices. In the experiments that were conducted without the use of the devices (no blowing-type or ventilationtype fan), when smoke accumulated in the gastrointestinal tract, it caused procedural difficulties, and all of the air in the gastrointestinal tract was aspirated along

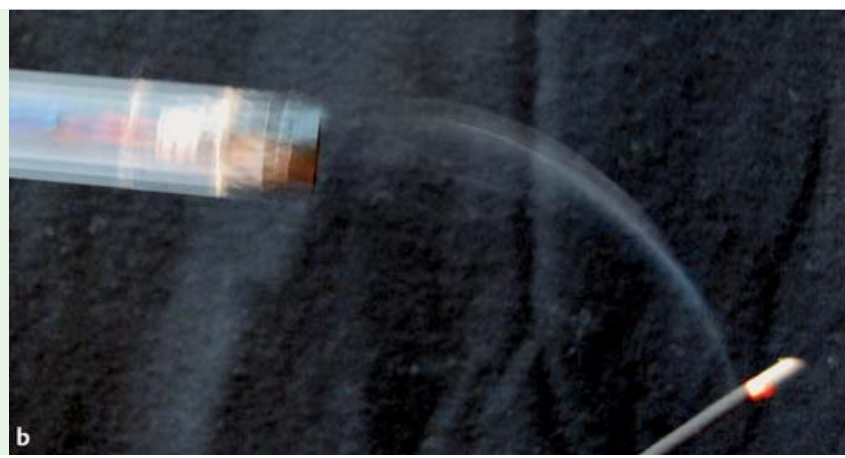

Fig. 3 Effect of the fan devices on the flow of smoke created with incense sticks: a blowing type; and $\mathbf{b}$ ventilation type. 


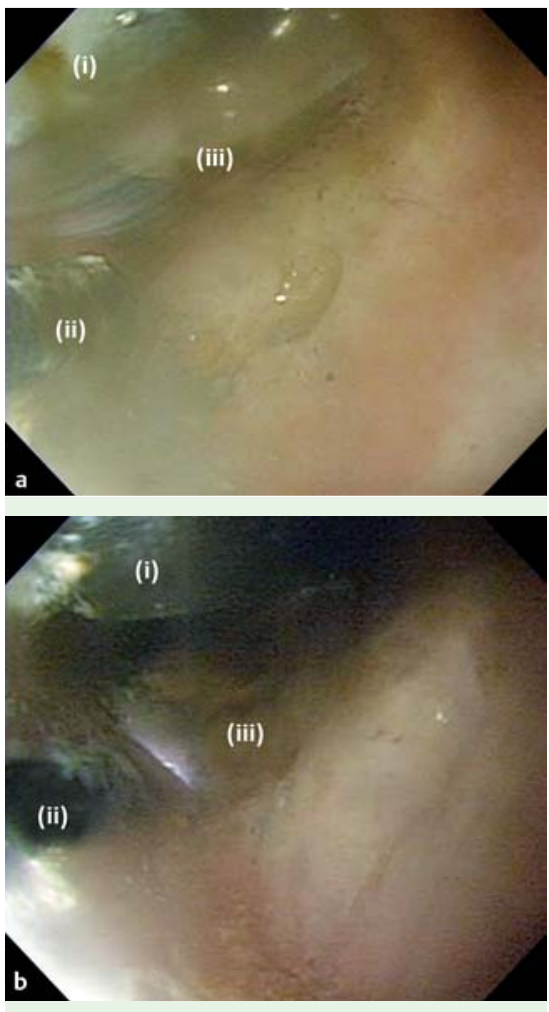

Fig. 4 Usefulness of the fan devices in endoscopic submucosal dissection (ESD). a A clear field of view has been obtained with the use of the blowing-type fan device, which has created a whirlpool of smoke surrounded by the blowing air. b Clear field of view obtained with the ventilation-type fan device. The smoke generated by the has been almost entirely removed with this device. (i), fan device; (ii), insulationtipped (IT) knife; (iii), clear field of view obtained by blowing or ventilation.

with the smoke and replaced with new air.

We used three resected porcine stomachs, each of which was cut into three similar sections. We carried out ESD three times for each experimental condition (without any device, with the fan-type device, and with the ventilation-type device) and noted the frequency of air replacements required during each procedure. The mean number of times the air was replaced was $4.33,2.67$, and 1.33 , for the procedures using no fan, or using the blowing-type or the ventilation-type fan, respectively. The mean time required for ESD was 42.33 minutes, 38.67 minutes, and 32.22 minutes, respectively. There was no significant differences in the mean size of the resected specimens among the three groups.

During endoscopic treatment, bleeding and mucus, and movement due to respiration are routinely encountered. In addition the patient may move or vomit. It is important that these are avoided or dealt with as soon as possible, without causing further hindrance for the endoscopic procedure being conducted. In the experimental ESD of the resected porcine stomachs, the two fan devices did not hinder the procedure.

Our findings indicate that the visual field can be improved during endoscopy by blowing away the air rather than changing the air volume, although occasional conventional aspiration is still required. A clear field of view renders endoscopic procedures simple and safe [1 -4].

The current fan devices cannot be used in patients as they are not sufficiently waterproof. After this problem has been resolved, further investigations will be required to evaluate the clinical utility of these devices. In conclusion, our experiment indicates that the fan devices may be useful in endoscopic treatment.

Endoscopy_UCTN_Code_TTT_1AO_2AG

\section{K. Kume}

Laboratory for Endoscopy, Third Department of Internal Medicine, University of Occupational and Environmental Health, Japan, School of Medicine, Kitakyusyu, Japan

\section{References}

1 Kume K, Yoshikawa I, Otsuki M. Endoscopic treatment of upper GI hemorrhage with a novel irrigating hood attached to the endoscope. Gastrointest Endosc 2003; 57: 732 735

2 Kume K, Yamasaki M, Yamasaki Tet al. Endoscopic hemostatic treatment under irrigation for upper GI hemorrhage: a comparison of one third and total circumference transparent end hoods. Gastrointest Endosc 2004; 59: 712 - 716

3 Kume K, Yamasaki M, Kanda $K$ et al. Endoscopic procedure under irrigation. Dig Endosc 2005; 17: 241 - 245

4 Kume K, Yamasaki M, Kubo $K$ et al. EMR of upper GI lesions when using a novel soft, irrigation, prelooped hood. Gastrointest Endosc 2004; 60: 124-128

\section{Bibliography}

DOI 10.1055/s-0029-1214923

Endoscopy 2009; 41: E236-E237

(c) Georg Thieme Verlag KG Stuttgart · New York . ISSN 0013-726X

\section{Corresponding author}

\section{K. Kume, MD, PhD}

Third Department of Internal Medicine

University of Occupational and Environmental

Health

Japan School of Medicine

1-1 Iseigaoka Yahatanishi-ku

Kitakyusyu 807-8555

Japan

Fax: +81-93-6920107

k-kume@med.uoeh-u.ac.jp 